\title{
Unique Characterization of Spatiotemporal Neural Network Activity
}

\author{
Sarita S. Deshpande, ${ }^{1,2,3 *}$ Graham Smith, ${ }^{3,4 *}$ Wim van Drongelen ${ }^{1,3,4 \dagger}$ \\ ${ }^{1}$ Committee on Neurobiology \\ ${ }^{2}$ Medical Scientist Training Program \\ ${ }^{3}$ Section of Pediatric Neurology \\ ${ }^{4}$ Committee on Computational Neuroscience \\ University of Chicago, Chicago, IL 60637, USA \\ ${ }^{*}$ These authors contributed equally. \\ ${ }^{\dagger}$ To whom correspondence should be addressed; E-mail: wvandron@peds.bsd.uchicago.edu.
}

We prove that three-spike motifs and phase relationships between frequency bands are essential to characterizing spatiotemporal neural activity and, in fact, sufficient. This follows from applying a theorem from optical science to show that all finite neural data have both unique triple (third-order) correlation and unique bispectrum. We simplify triple correlation by classifying three-spike motifs into motif-classes according to their sequencing, which can embody well-studied neural properties such as synchrony, feedback, feedforward, convergence, and divergence. Summing triple correlations within these classes yields a summary revealing structure underlying the neural network data. This simple approach demonstrates the power of complete statistical representations of neural activity. Since these representations generalize across recording modalities, we present our analysis as a potential avenue for investi- 


\section{gation throughout the field.}

Researchers use increasingly diverse modalities to record neuronal activity, and many more methods to analyse and parse patterns from these data. Even so, the raw representation of network spiking activity has been the same for decades: the raster plot (Fig. 1A). Analysis of raster plots tends to be based on statistics of time bins or at most their pairwise interactions (e.g. cross-correlation (1-3)). As valuable as such analyses have been, they must inevitably fail to capture some aspects of network activity: no combination of first- and second-order statistics can reconstruct activity. In a system as complex as the brain, with billions of recurrent interactions, researchers have assumed that reconstructing activity would require adding higherorder interactions (4), perhaps ad infinitum. In fact, here we prove that third-order interactions are sufficient to completely represent spatiotemporal neural activity.

Using only third-order statistics of the raster, we derive a theoretically complete correlation analysis, which implicitly provides fundamental building blocks of brain activity. To do this, we interpret the spike raster as an image in order to apply the Triple Correlation Uniqueness (TCU) theorem. The TCU theorem was first used in optical science, introduced by Bartelt et al. (5), then refined and named by Yellott and Iverson (6). Yellott (7) used it to state a corrected version of the then-disproven Julesz conjecture $(8,9)$. Julesz had conjectured that the brain cannot spontaneously discriminate between images with identical second-order statistics. With the TCU theorem, Yellott proved that any human observer is unable to distinguish between images with identical third-order statistics, for the simple reason that such images are identical (up to translation).

Here, we bring the TCU theorem into the heart of neuroscience to characterize spike rasters and thus to characterize neural network activity patterns. We begin by describing triple correlation $\left(c_{T}\right)$ and briefly summarizing the proof of the unique correspondence of $c_{T}$ to its associated image. Then, we examine triple correlation in terms of its basic units, which encode the rela- 
tionship among three spikes. We classify these unit interactions as spike sequences, which we use to interpret underlying structure in example simulations.

Unlike pairwise correlation (function of the lag between two spikes), triple correlation characterizes three-way interactions as a function of two lags among three spikes (Fig 1B). For a spatiotemporal raster, $r(n, t)$ ( $n$, space; $t$, time), $c_{T}$ is a function of four variables: $c_{T}\left(n_{1}, t_{1}, n_{2}, t_{2}\right)=\left\langle r(n, t) r\left(n+n_{1}, t+t_{1}\right) r\left(n+n_{2}, t+t_{2}\right)\right\rangle_{n, t}$. The operation $\langle\ldots\rangle_{n, t}$ computes the average over all bins $(n, t)$ in the raster. The $\ldots$ is 1 when $(n, t)$ is part of a spiking triplet with lags $\left(n_{1}, t_{1}, n_{2}, t_{2}\right)$, which we call a lag-motif, and 0 otherwise.

The TCU theorem can be applied to any finite bounded dataset by interpreting that dataset as an image, so we can apply it to a spike raster (proof in Supplemental Materials). As a result, third-order statistics entirely characterize neural spiking activity, i.e. spiking activity can be recovered in its entirety from triple correlation (see "Reconstruction Algorithms for Finite Images" in (6)). The triple correlation's fundamental units are the prevalences of threespike motifs, and therefore three-spike motifs are building blocks of network spiking activity. Simultaneously, the same uniqueness of the bispectrum proves the fundamental importance of phase and amplitude of inter-frequency-band relationships between spike trains.

From this complete statistical representation, we extracted meaningful global patterns among the raster's spikes, and hence implicitly among the network's neurons. We did this by classifying the lag-motifs - the basic unit of triple correlation-according to their relative spiking sequence between neurons. First, we applied the most essential distinction, that on the basis of lag's sign $(-, 0,+)$, to obtain 169 lag-sign motifs (Tables S1, S2). We then reduced these 169 lag-sign motifs to 14 motif-classes (Fig. 1, Table S2) on the basis of distinct spike sequences implied by the lag-signs, which can be grouped through certain commonalities. For example, due to the way the lag-sign motifs are labelled, many are functionally identical (e.g. lag-sign motifs 8.1.1 and 8.4.1, Table S2). Additionally, since we did not order the spatial dimension in 
the raster, there is spatial symmetry (e.g. lag-sign motifs 7.1 and 7.3, Table S2). See supplement for a full accounting.

To evaluate our classification, we simulated neural spiking activity in a network of 150 neurons over 150 time steps, represented as a $150 \times 150$ raster with each spike a single black pixel. In our example, we implicitly assume that a 15 bin lag window in either dimension encompasses all correlations of interest. Thus, we calculated a $15 \times 15 \times 15 \times 15$ triple correlation, i.e. the prevalences of 50,625 lag-motifs, which we then classified into 14 motif-classes of spike sequences, as above (Fig. 1). Note that if the lag window must be increased, the number of lag-motifs scales quartically with number of lags, while the number of motif-classes remain constant.

To illustrate our classification on a network with simple structure, we simulated a network spike raster with synchronous, periodic firing at a frequency, $f=0.08$ arbitrary units (AU) (Fig. 2A). From that raster, we determined each motif-class' actual contribution by summing the raster's triple correlation across all lag-motifs in that motif-class (Fig. 2B). For the same sized raster, we calculated two additional contributions: the expected noise contribution from a rate-matched raster with random spiking (Fig. 2C-D), and the maximum potential contribution from a fully spiking raster (Fig. 2E-F). We calculated the actual-to-potential contribution ratio (A/P; Fig. 2G) to control for the motif-classes' differing theoretical combinatorial maxima; we calculated the actual-to-noise contribution ratio (A/N; Fig. 2H) to control for the motif-classes' expected contributions due to pure chance. Intuitively, we expect these ratios to reflect structure in the spike propagation of the underlying network. In general, if an observed spike sequence is associated with network function, the motif-classes in Fig. 1C embody well-known neuronal processing properties, as summarized in Table 1. Motif-class I, which merely reflects the spike rate, is relatively high (albeit $<1$ ). Indeed in our synchronous simulation we see that motifclasses IV and V, which correspond purely to neural synchrony, are closest to their maximum 
potential (Fig. 2G) and are highest above noise (Fig. 2H).

From our analyses, we found that the large potential contributors to the triple correlation include feedforward, divergence, convergence and to a lesser extent, feedback. In contrast, individual neuronal activity, synchrony and spike propagation contribute to many or all motifclasses (Motif-Classes, Table 1), but their pure potential contributions are modest (Characteristic Classes, Table 1), i.e. all $\leq 1 \%$.

We further explored examples of other network structures using these metrics. First we examined a network with faster synchronous firing (Fig. 3A), which reflects similar results to those of the slower synchronous firing. The motif-classes found more often than chance are precisely those with an element of synchrony (IV, V, VII, VIII, XII, XIII; motif-classes with vertical dashed brown lines in Fig. 1). Second, we phase shifted the synchronous activity (Fig. 3B), which had the expected result of slightly reducing the $\mathrm{A} / \mathrm{N}$ of purely synchronous motif-classes (IV, V) and slightly increasing A/N of spike propagation (VI) and related motif-classes (IX, X, XI). Finally, we added noise ( $S N R=0 \mathrm{~dB}$, Fig. 3C), which uniformly moved A/N towards 1 for all motif-classes, as expected, while maintaining $>1$ values for synchronous motif-classes. We confirmed expected quantification of exemplars of each motif-class via further test simulations (Fig. S1).

Our particular choice of classification is simply one demonstration of the power of having triple correlation as a unique statistical representation. Note that this demonstration is natural because it is purely derived from spike sequence, due only to the unidirectionality of time. However, the essential result is the underlying three-spike motif building blocks, which are fundamental to the raster's triple correlation and hence to spatiotemporal neural activity. Similarly, phase and amplitude inter-frequency-band relationships between neurons are fundamental to the bispectrum, which in constituting an equivalent unique representation, proves the fundamental importance of inter- and intra-neuronal inter-band analyses. 
Both three-spike motifs and inter-band analyses are important topics of investigation in neuroscience (e.g. (10-15)). Here, we have provided a direct avenue for these investigations and proven the fundamental importance of these topics to any spatiotemporal neural data. From this, the clinical applications abound (e.g. (15-18)). We chose to simulate spiking activity in one spatial dimension as a simple illustration of this approach. However, our methodology can extend beyond spike rasters to higher spatial dimensions or to continuous-valued signals, such as multi-electrode local field potential or electroencephalogram recordings. Triple correlation and bispectrum uniqueness holds promise as an innovative approach to analyzing spatiotemporal neural activity with applications that span the breadth of recording modalities.

\section{References}

1. T. Tchumatchenko, T. Geisel, M. Volgushev, F. Wolf, Front. Neurosci. 5 (2011).

2. E. Schneidman, M. J. Berry, R. Segev, W. Bialek, Nature 440, 1007 (2006).

3. M. Jiang, X. Gao, H. An, H. Li, B. Sun, Sci. Rep. 7, 10486 (2017).

4. S. Yu, et al., J. Neurosci. 31, 17514 (2011).

5. H. Bartelt, A. W. Lohmann, B. Wirnitzer, Applied Optics 23, 3121 (1984).

6. J. I. Yellott, G. J. Iverson, J. Opt. Soc. Am. A 9, 388 (1992).

7. J. I. Yellott, J. Opt. Soc. Am. A 10, 777 (1993).

8. B. Julesz, IRE Trans. Info. Theory 8, 84 (1962).

9. B. Julesz, E. N. Gilbert, L. A. Shepp, H. L. Frisch, Perception 2, 391 (1973).

10. S. Jovanović, S. Rotter, PLoS Comput. Biol. 12, e1004963 (2016). 
11. R. Milo, et al., Science 298, 824 (2002).

12. K. Bojanek, Y. Zhu, J. MacLean, PLoS Comput. Biol. 16, e1007409 (2020).

13. J. B. Dechery, J. N. MacLean, PLoS Comput. Biol. 14, e1006153 (2018).

14. O. Sporns, R. Kötter, PLoS Biol. 2, e369 (2004).

15. N. A. Jansen, et al., J. Neurosci. 41, 524 (2021).

16. S. V. Canavan, et al., Validating Neuro-Computational Models of Neurological and Psychiatric Disorders, B. S. Bhattacharya, F. N. Chowdhury, eds., Springer Series in Computational Neuroscience (Springer International Publishing, Cham, 2015), pp. 293-312.

17. M. Goodfellow, et al., Sci. Rep. 6, 29215 (2016).

18. J. Hebbink, H. Meijer, G. Huiskamp, S. van Gils, F. Leijten, Epilepsia 58, e147 (2017).

19. Z. Moszner, aequationes mathematicae 21, 20 (1980).

20. W. van Drongelen, Signal processing for neuroscientists: Introduction to the analysis of physiological signals. (Elsevier/Academic Press, 2018), second edn.

21. W. Feller, An introduction to probability theory and its applications, (Wiley, Oxford, England, 1950).

\section{Acknowledgments}

We would like to thank Drs. J. MacLean and S.A. van Gils for thoughtful discussion and feedback. S.S.D. is supported by University of Chicago MSTP Training Grant T32GM007281. G.S. is supported by the Pritzker Endowment for the Neurosciences. 


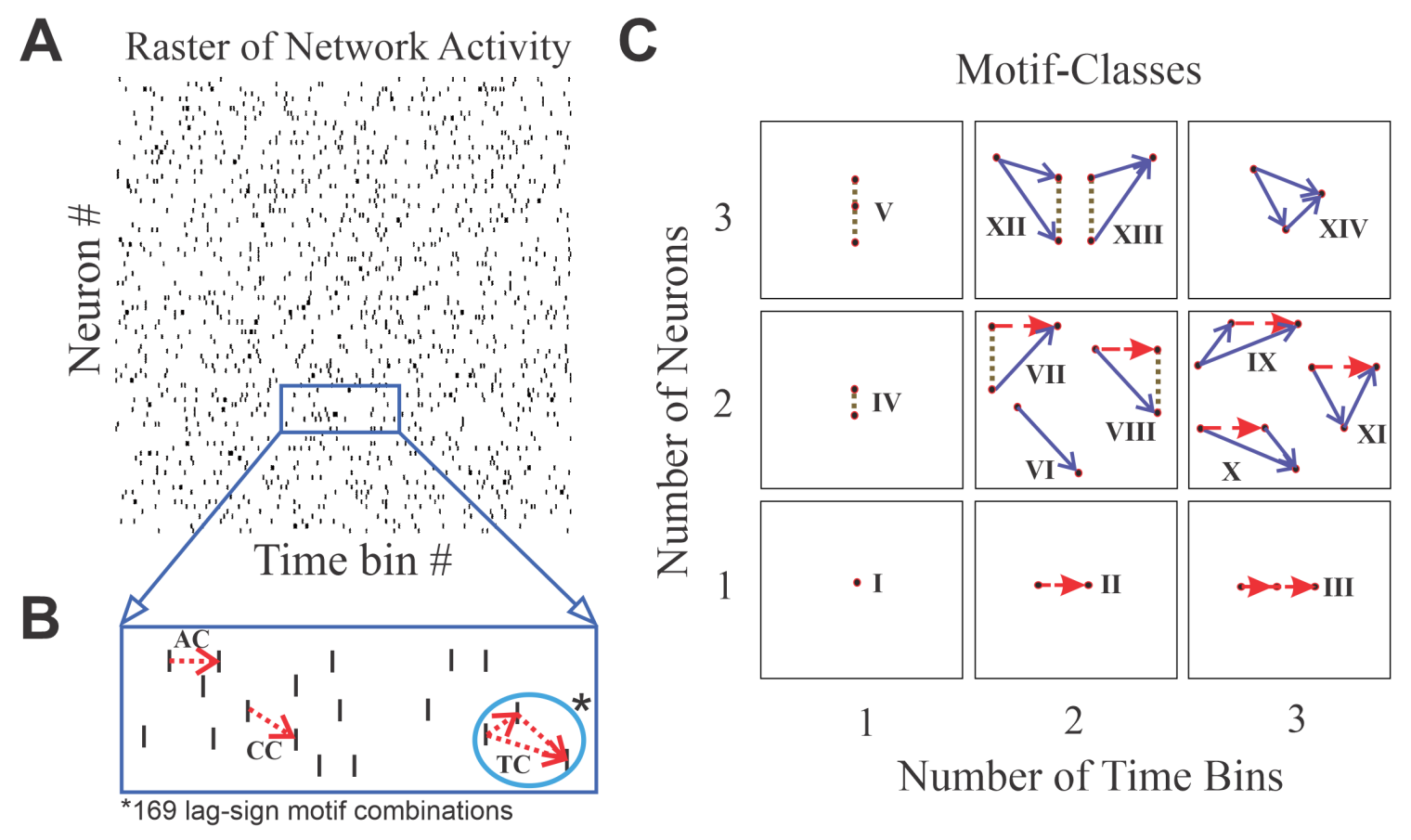

Fig. 1. Application of the $\mathbf{1 4}$ motif-classes to the spike raster. For a given raster (Panel A), second-order correlations can relate activity within a neuron (auto-correlation, AC; Panel B) or between neurons (cross-correlation, CC; Panel B). Triple correlation (TC; Panel B) relates three-spike motifs with up to two temporal and two spatial lags, a lag-motif. We classify these lag-motifs into 14 motif-classes by the triplet's spike sequence (Panel C, Table S2). Absence of a time-lag between different nodes indicates synchronous firing by multiple neurons and is represented by a vertical brown stippled line without an arrow head (e.g. IV). Absence of spatial-lag with a non-zero time-lag indicates repeated spiking within a single neuron and is represented by a horizontal red dashed line with an arrow head (e.g. II). Non-zero lags in both time and space are indicated by solid blue arrows (e.g. VI).

Table 1. Neural processing properties from Fig. 1 and their potential contributions to triple correlation. Neural processing properties (Property, column 1) can be implemented by multiple motif-classes (column 2). Fewer motif-classes can purely implement these properties (Characteristic Classes, Char., column 3). We calculate the potential contribution of each prop- 
erty as the sum of the potential contributions (Fig. 2) of its characteristic classes (Pot., column 4). Note that the potential contributions do not add up to $100 \%$, both because there is overlap in the characteristic classes and because some motif-classes are not characteristic of any property. Motif-class XIV is unique in that it can purely implement any of three properties. Note that some properties imply others, e.g. feedback includes spike propagation, so XI includes both feedback and spike propagation, but we still say it is the pure feedback motif.

\begin{tabular}{c|ccc} 
Property & Motif-Classes & Char. & Pot. \\
\hline Spike Rate & (all) & I & $<0.01 \%$ \\
Local Dynamics & II, III, VII, VIII, IX, X, XI & II, III & $0.4 \%$ \\
Synchrony & IV, V, VII, VIII, XII, XIII & IV, V & $0.4 \%$ \\
Spike Propagation & VI, VII, VIII, IX, X, XI, XII, XIII, XIV & VI & $1 \%$ \\
Feedback & XI & XI & $5.0 \%$ \\
Feedforward & XIV & XIV $^{*}$ & $65 \%$ \\
Divergence & XII, XIV & XII, XIV $^{*}$ & $73 \%$ \\
Convergence & XIII, XIV & XIII, XIV $^{*}$ & $73 \%$
\end{tabular}


A

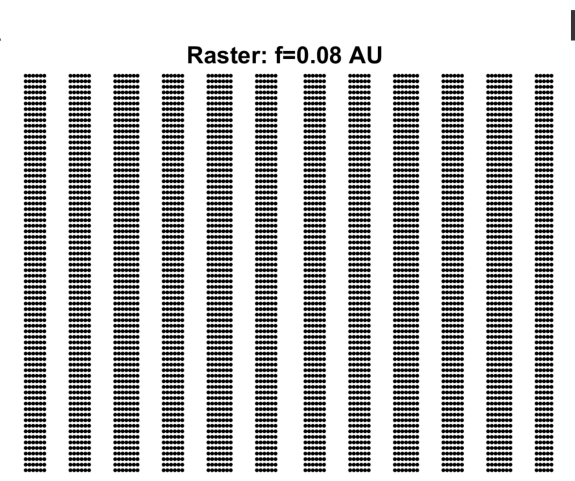

C

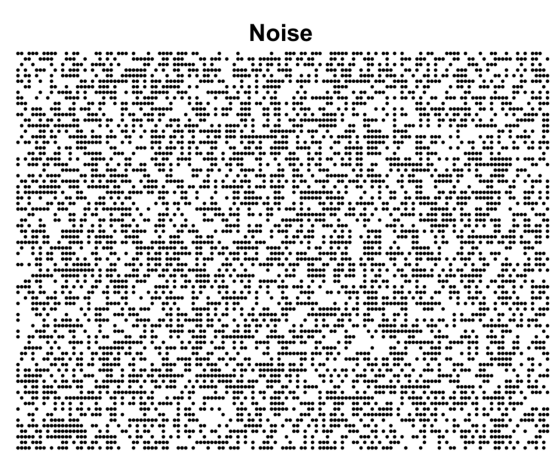

E
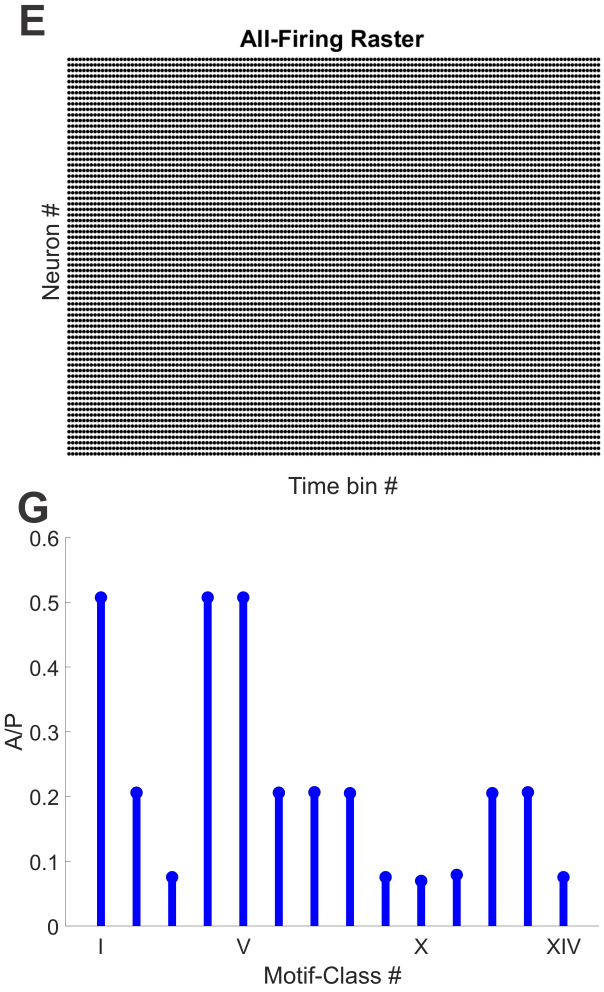

B

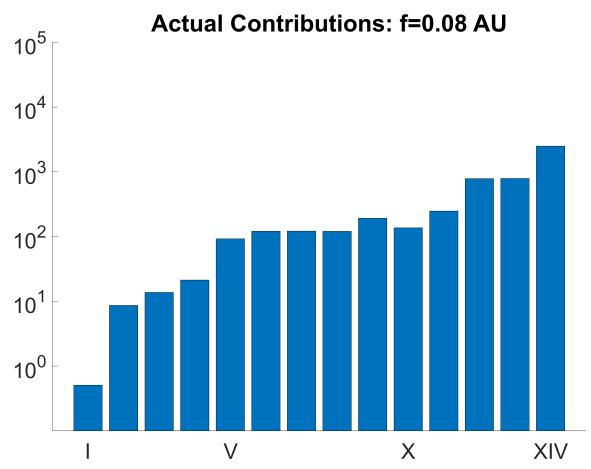

D

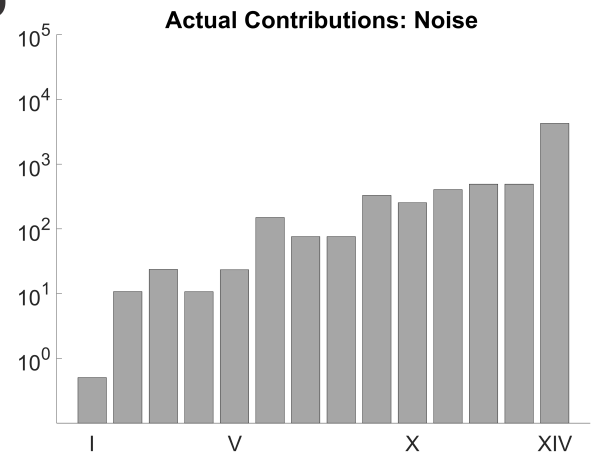

$F$

Potential Contributions

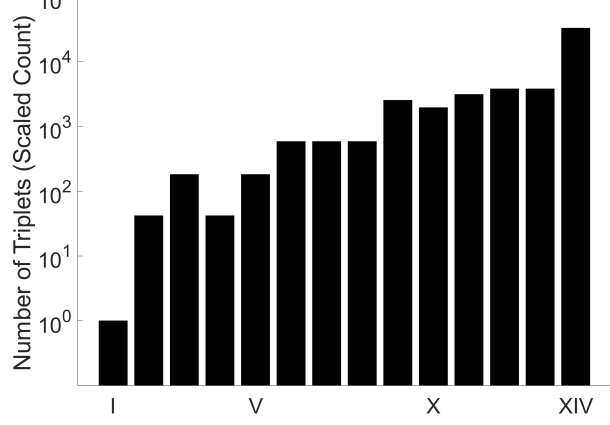

H

Motif-Class \#

5

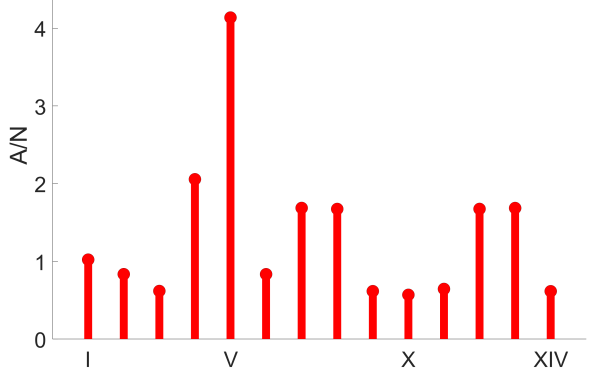


Fig. 2. Potential contribution of each motif-class to the raster's triple correlation. A) The $150 \times 150$ spike raster plot has a frequency of 0.08 AU. B) The actual contributions of the 14 motif-classes for a periodic raster pattern shown in Panel A. C) Noise raster with activity matched to Panel A. D) The actual contributions for the raster shown in Panel C. E) The allfiring raster. F) The contribution for each of the 14 motif-classes for the raster in Panel $\mathrm{E}$ to the triple correlation of a $150 \times 150$ raster plot, which we call the potential contribution, which is the maximal possible contribution since it is based on a spike from every neuron\# at every time bin. G) The ratios between the actual-and-potential contributions (A/P) is calculated by taking the ratios between Panels B and F. H) The ratios between the actual-and-noise contributions $(\mathrm{A} / \mathrm{N})$ is calculated by taking the ratios between Panels B and D. 
A

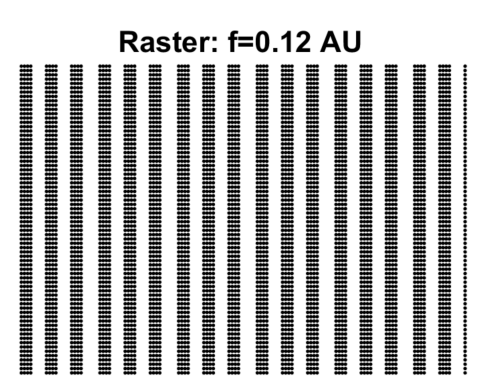

B

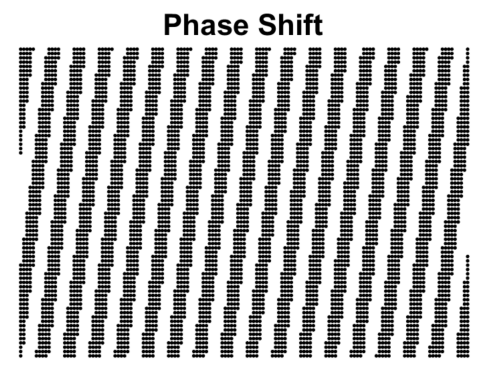

$\begin{gathered}100 \\ \text { spikes }\end{gathered} \mid$
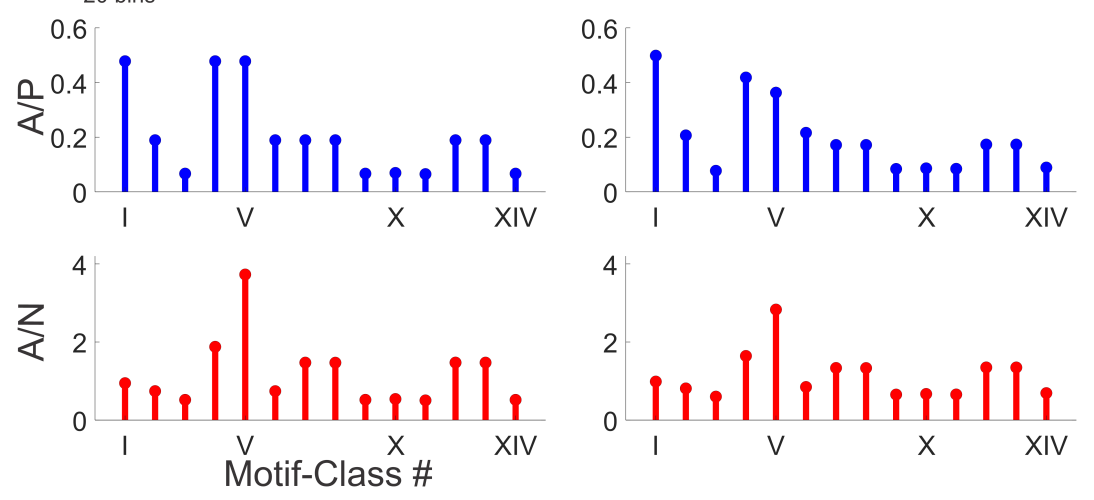

C SNR $=0 \mathrm{~dB}$
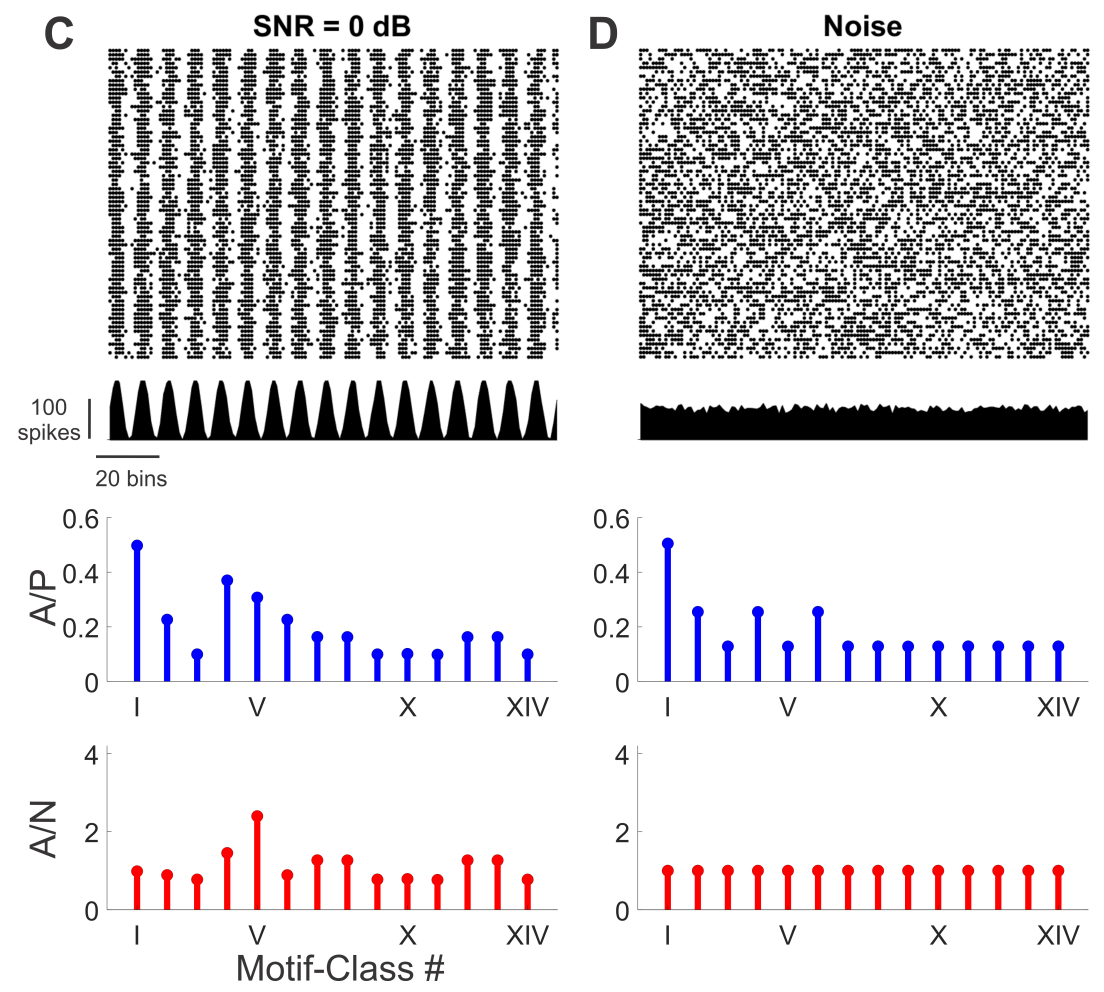
Fig. 3. Dependence of motif-class contribution on network structure. In each panel, the raster plot of multi-unit spiking activity, the sum of the raster's activity, and the relative contributions are shown for each of the 14 motif-classes. The ratios of actual-to-potential contributions $(\mathrm{A} / \mathrm{P})$ are shown in blue and the ratios of actual-to-noise contributions $(\mathrm{A} / \mathrm{N})$ are shown in red. A) Frequency $f=0.12$ AU. B) Same as Panel A with phase shift. C) Same as in panel A with added noise $\mathrm{SNR}=0 \mathrm{~dB}$. D) Noise only. 